Dig Dis 1996;14:393-394

\title{
Subject Index Vol. 14, 1996
}

Vol. 14, Supplement 1, has its own Subject Index

Acute pancreatitis 83 Angiodysplasia 345 Angioplasty 99 Antibodies 192 Antireflux surgery 169 Ascites 145 Autoimmune hepatitis 157

Balloon distension 276 Bandligation 201,356 Barostat 276 Barrett’s esophagus 213 Bile duct injury 371

- $\quad$ leak 371

Biliary stricture 180

Budd-Chiari syndrome 99

Calcium 119

Carcinoembryonic antigen 5 Cholangiocarcinoma 180 Cholangiography 371 Cholecystokinin receptors 334 Choledocholithiasis 371 Chronic liver disease 73

pancreatitis 83 Colon 228

cancer 5, 119 Colonoscopy 228 Colorectal cancer screening 132 Congestive gastropathy 27,258

Cryoglobulinemia 157 Cytotoxin 289

Diet 43

Dietary fiber 43 Diuretics 145 Dysphagia 323 Dysplasia 213

Endoscopic retrograde cholangio-pancreatography 371

- $\quad$ sphincterotomy 371

Endoscopy 228, 356

End-stage liver disease 316

Esophageal varices, therapy

356

Esophagogastroduodenoscopy 228 Esophagus 201,323

Flexible sigmoidoscopy 228 Follow-up, colorectal cancer 5 Functional dyspepsia 276 Functional gastro-intestinal disorders 276

Gastric atrophy 27 Gastrin receptors 334 Gastritis 289 Gastritis/duodenitis 27 Gastroduodenal

dyspepsia 27 Gastroesophageal reflux disease

169 Gastrointestinal bleeding 228

hemorrhage 201,258

tract 14 Genetic disease 304 Glandular epithelial neoplasms

245 Glomerulonephritis 157

Helicobacterpylori 27, 289 Hemochromatosis 304 -, hereditary 316 Hepatic hydrothorax 73

Hepatitis C virus 157 Hepatopulmonary syndrome 73 HLA locus 304 Hypercoagulable state(s) 99, 382

Idiopathic dyspepsia 27 IgA 192

Immunization 192 Immunoglobulins 192 Intestine, adaptation 59 Intraoperative enteroscopy 345

Iron overload 304 Irritable bowel syndrome 276 Ischemic bowel 382

Laparoscopic cholecystectomy 14, 371 
- $\quad$ surgery 14

Laparoscopy 169

Liver 304

Liver transplantation 145,316 Lung disease 73 Lymphocytes 192 Lymphoma 289

Lymphoplasmacytic gastritis 27

Management 213 Molecular mechanism 59 Myocardial infarction 228

Neutrophilic gastritis 27 Nissen fundoplication 169 Non-cardiac chest pain 276 Nonsteroidal anti-inflammatory

drugs (NSAIDs) 119 Nonulcer dyspepsia 27 Nutrient 59 Nutrition 43

Occult gastrointestinal bleeding 345

Pancreatitis 83, 371 Panendoscopy 228 Paracentesis 145 Peptides, thymic 362 Peyer's patches

192 Porphyria cutanea tarda 157 Portacaval shunts 99 Portal hypertension 201, 258

- $\quad$ hypertensive gastropathy 258

Postoperative endoscopy 228

Pregnancy 228

Prevention, cancer 43 Prognosis, colorectal cancer 5 ProteinS 382

Pulmonary hypertension 73 Push enteroscopy 345

Receptor states 334 Recurrence, colorectal cancer 5

393

Reinfusion, concentrated ascites Stomach 228 Transjugular intrahepatic porto-

145 Stool testing $132 \quad$ systemic shunt 145

Resection, bowel 59 Suction sclerotherapy, esophageal

Risk factors $83 \quad$ varices 356 Urease 289

Surgical innovation 14

Schatzki’s ring 323 Surveillance $213 \quad$ Vaccines 192

Sclerosing cholangitis 180 Varices 201

Sclerotherapy 201 Therapy 43 Venous thrombosis 99

Small intestinal neoplasms 245 Thymic extracts 362 Viral chronic hepatitis 362

Sonde enteroscopy $345 \quad$ Thymosin 362 Visceral perception 276

Spiro syndrome 27 Thyroiditis $157 \quad$ Vitamins 119

394

Subject Index 